\title{
Glutamine:fructose-6-phosphate aminotransferase enzyme activity is necessary for the induction of TGF- $\beta 1$ and fibronectin expression in mesangial cells
}

\author{
C. Weigert, U. Friess, K. Brodbeck, H. U. Häring, E. D. Schleicher \\ Department of Internal Medicine, Division of Endocrinology, Metabolism and Pathobiochemistry, University of Tübingen, \\ Tübingen, Germany
}

\begin{abstract}
Aims/hypothesis. Increased flux through the hexosamine biosynthetic pathway with glutamine:fructose-6phosphate aminotransferase (GFAT) as a rate-limiting enzyme has been linked to the enhanced bioactivity of the prosclerotic cytokine TGF- $\beta 1$, a key mediator in the development of diabetic nephropathy and possibly other diabetic angiopathies. In this study we investigated the effect of enhanced expression of wild-type GFAT and two enzymatically inactive GFAT mutants on TGF- $\beta 1$ synthesis in mesangial cells.

Methods. Mutated human GFAT expression vectors were prepared by PCR-site directed mutagenesis. Wild-type and mutated vectors were transfected into human embryonic kidney 293 cells and mesangial cells and GFAT enzyme activity was assessed by formation of glucosamine-6-phosphate. Production of TGF- $\beta 1$ and fibronectin protein was examined by ELISA.
\end{abstract}

Results. Mutation of histidine 577 or lysine 676 to alanine led to a complete loss of GFAT enzyme activity. An increased concentration of wild-type GFAT in mesangial cells enhanced both TGF- $\beta 1$ and fibronectin production 1.5 -fold, while mesangial cells transfected with the mutated GFAT constructs showed no effect.

Conclusion/interpretation. The data indicate that the hexosamine pathway-mediated induction of TGF- $\beta 1$ synthesis in mesangial cells is dependent on GFAT enzyme activity. Our results add to previous observations showing that the hexosamine pathway could increase the transcriptional activity of nuclear proteins leading to enhanced cytokine synthesis. [Diabetologia (2003) 46:852-855]

Keywords Diabetic nephropathy, GFAT overexpression, TGF- $\beta 1$, fibronectin, mesangial cells.
Glutamine:fructose-6-phosphate aminotransferase (GFAT) is the rate-limiting enzyme of the hexosamine biosynthetic pathway catalyzing the conversion

Received: 19 September 2002 / Revised: 21 November 2002 Published online: 11 June 2003

(C) Springer-Verlag 2003

Corresponding author: Dr. E. D. Schleicher, Department of Internal Medicine, Division of Endocrinology, Metabolism and Pathobiochemistry, University of Tübingen, Otfried-MüllerStraße 10, 72076 Tübingen, Germany

E-mail: enschlei@med.uni-tuebingen.de

Abbreviations: GFAT, glutamine:fructose-6-phosphate aminotransferase; GlcN-6-P, glucosamine-6-phosphate; HEK 293 cells, human embryonic kidney cells; RP-HPLC, reversedphase high performance liquid chromatography; UDP-GlcNAc, uridine-5' -diphosphate-N-acetylglucosamine. of fructose-6-phosphate to glucosamine-6-phosphate $(\mathrm{GlcN}-6-\mathrm{P})$ [1]. The product GlcN-6-P is very rapidly further converted to uridine-5'-diphosphate-N-acetylglucosamine, a substrate for the O-GlcNAc-modification of cytosolic and nuclear proteins possibly leading to altered transcription factor activity [2].

Recent studies have indicated that the hexosamine pathway could have regulatory functions as a nutrientsensing system [3, 4], thereby linking energy intake and energy expenditure [5]. Moreover, the hexosamine pathway has also been implicated in the development of diabetes-associated macrovascular complications [6] and glomerulosclerosis [7]. In particular, the hexosamine pathway mediates the adverse effects of hyperglycaemia through an induced action of the prosclerotic cytokine TGF- $\beta 1$ leading to the thicken- 
ing of glomerular and tubular basement membranes $[7,8]$. Inhibition of the hexosamine pathway by the glutamine-analogue azaserine prevented the high glucose-induced TGF- $\beta 1$ synthesis and bioactivity and the enhanced expression of matrix proteins in glomerular mesangial cells [7]. However, azaserine also influences the numerous glutamine-dependent reactions in metabolism thus possibly exerting toxic effects.

To evaluate the role of GFAT in diabetic glomerulosclerosis we examined the effect of enhanced GFAT expression in cultured mesangial cells, since glomerular mesangial cells are important effectors in the early events in the development of diabetic nephropathy. Based on our recent data showing that an increase in GFAT wild-type protein increases TGF- $\beta 1$ and fibronectin protein production in NIH 3T3 fibroblasts [9] we developed two enzymatically inactive GFAT mutants to verify the importance of a functionally active GFAT protein for TGF- $\beta 1$ induction.

\section{Materials and methods}

Materials. Mouse mesangial cell line SV40 MES and HEK 293 cells were obtained from ATCC (Manassas, USA). Oligonucleotides were synthesized by Life Technologies (Karlsruhe, Germany). Cell culture media, supplements, Ultroser and foetal calf serum were from Gibco (Eggenstein, Germany). Azaserine and 6-diazo-5-oxonorleucine were from Sigma (Munich, Germany). Production and characterization of the antibody against GFAT was described recently [10].

Cell culture. Mouse SV40 mesangial cells and HEK 293 cells were grown in DMEM containing $25 \mathrm{mmol} / 1$ glucose, $10 \%$ foetal calf serum, $1 \mathrm{mmol} / \mathrm{l}$ sodium pyruvate, $4 \mathrm{mmol} / \mathrm{l}$ glutamine, nonessential amino acids, $100 \mathrm{U} / \mathrm{ml}$ penicillin and $100 \mu \mathrm{g} / \mathrm{ml}$ streptomycin. For experiments, mesangial cells were cultured in $5.5 \mathrm{mmol} / \mathrm{l}$ glucose and in the same medium but without serum for determination of TGF- $\beta 1$ and fibronectin.

Preparation of GFAT expression vectors. A full-length human GFAT cDNA was generated [9] and cloned into the expression vector pcDNA3.1 (Invitrogen, Inchinnan, Scotland). The PCRproduct was verified by sequencing and showed identity to human GFAT (GenBank accession number M90516). For mutation of amino acid position 577 histidine to alanine and of amino acid position 676 lysine to alanine base substitutions were made by oligonucleotide-mediated mutagenesis. The mutagenic upstream primers used are ala577/1: GTGGTGAATTGAAAGCTGGCCCTCTGGCTTTGG and ala676/1: CCACGGAATCTTGCCGCATCTGTGACTGTAGAG with the wild-type GFAT expression vector as template. Positive clones were verified by sequencing.

Transfection methods. HEK 293 cells were transfected using $\mathrm{Ca}_{3}\left(\mathrm{PO}_{4}\right)_{2}$-DNA-coprecipitation-method. Mesangial cells were transfected with Superfect (with an average transfection efficiency of $10 \%$ ).

Assay of GFAT enzyme activity. GFAT enzyme activity was measured by quantification of GlcN-6-P according to: fructose-6-phosphate + glutamine $\rightarrow$ glucosamine-6-phosphate + glutamate. Previously published methods were modified and optimized for the analysis in small volumes of cell lysates using a programmable autosampler for pre-column o-phtaldialdehyde derivatisation, RP-HPLC for separation and fluorescence for detection. For identification and preparation of standard curves GlcN-6-P was used. Briefly, cells were scraped into extraction buffer, sonicated on ice and centrifuged. We incubated two $100 \mu$ l-aliquots of a once-thawed lysate with reaction buffer containing $10 \mathrm{mmol} / \mathrm{l}$ fructose-6-phosphate and $10 \mathrm{mmol} / \mathrm{l}$ glutamine for $0 \mathrm{~min}$ (blank) and $45 \mathrm{~min}$ at $37^{\circ}$, stopped by denaturation $\left(100^{\circ}\right.$ for $\left.3 \mathrm{~min}\right)$ und analyzed by HPLC. A crude preparation of overexpressed GFAT protein from drosophila obtained from H.-R. Graack, Berlin, was used as positive control. The CV for the complete assay (enzyme reaction/derivatisation/HPLC-analysis) was less than $15 \%$.

Western blotting. Cellular extracts of GFAT transfectants or controls were applied to sodium dodecyl sulfate (SDS) gel electrophoresis and Western blotting was carried out [9].

Determination of TGF- $\beta 1$, fibronectin and DNA concentrations. Determination of total TGF- $\beta 1$ protein was done with the Quantikine Immunoassay (R\&D Systems, Minneapolis, USA). Fibronectin was measured by the Quantimatrix human fibronectin ELISA kit from Chemicon International (Temecula, Calif., USA). DNA was measured by fluorometry with bisbenzimidazol [7].

Statistical analysis. Results shown are derived from at least three independent experiments. Means \pm SEM were calculated and groups of data were compared using Student's $t$ test. A $p$ value of less than 0.05 was considered statistically significant.

\section{Results}

Mutation of histidine-577 or lysine-676 to alanine in GFAT protein abolished enzyme activity. To evaluate the effect of enhanced GFAT enzyme activity we mutated histidine 577 and lysine 676 of the GFAT protein to alanine, because either amino acid has been postulated to play an essential role in the conversion of fructose-6-phosphate to glucosamine-6-phosphate. Wildtype and the mutated GFAT sequences were transfected into HEK 293 cells, a cell line which provides very high expression levels. A high and comparable GFAT protein expression level was obtained as assessed by Western blotting (Fig. 1A). Measurement of GFAT activity in the cell lysates showed a fifteen-fold increase of enzyme activity in the wild-type GFAT transfected cells, while transfection of the empty control expression vector or of the mutated GFAT expression vectors yielded a similiar low enzyme activity (Fig. 1B), probably representing the endogenous activity. These data indicate that both mutations resulted in essentially complete loss of GFAT enzyme activity.

Overexpression of GFAT in mesangial cells induces $T G F-\beta 1$ and fibronectin production. To study the effect of enhanced GFAT activity on TGF- $\beta 1$ synthesis in mesangial cells we carried out transient transfection experiments using the wild-type and mutated GFAT expression vectors described above. Western blotting 
A

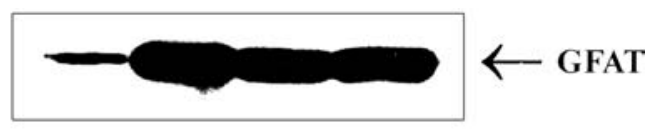

B

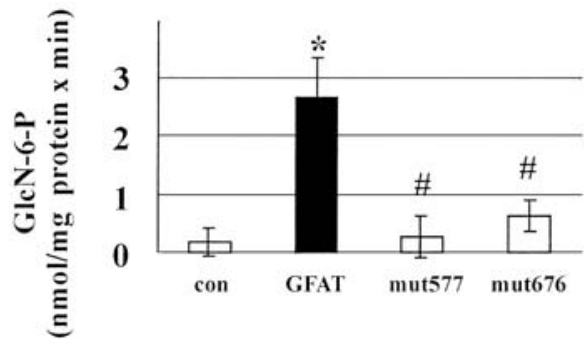

Fig. 1A, B. Effect of mutation of histidine 577 (mut 577) or lysine 676 (mut 676) to alanine of GFAT on GFAT enzyme activity. (A) Western blotting of the cellular extracts using anti-GFAT antibody. Detected GFAT protein is marked by an arrow. (B) Mutated and wild-type expression vectors were transfected into HEK 293 cells and GFAT enzyme activity was measured in the cellular extracts $40 \mathrm{~h}$ after transfection. Enzyme activities were expressed as amount of produced GlcN-6-P nmol $\times \mathrm{mg}^{-1}$ protein $\times \mathrm{min}^{-1}$ assessed by HPLC. Three different independent experiments were carried out. Results shown are means \pm SEM. $* p<0.05$ wild-type vs. control, $\# p<0.05$ mutant vs. wild-type

showed that GFAT protein expression was increased approximately threefold (data not shown). Transfection of the wild-type GFAT expression vector into mesangial cells led to a 3.9-fold induction of GFAT enzyme activity compared to cells transfected with the empty control vector $(0.77 \pm 0.38$ vs. $2.98 \pm 0.4 \mathrm{GlcN}$ 6-P nmol $\times \mathrm{mg}^{-1}$ protein $\left.\times \mathrm{min}^{-1}\right)$.

Determination of the amount of secreted TGF- $\beta 1$ protein in the supernatant of the transfected cells indicated that only overexpression of the enzymatically active wild-type GFAT protein in mesangial cells enhanced TGF- $\beta 1$ synthesis 1.5 -fold, while transfection of the mutated GFAT plasmids had no effect (Fig. 2A). No difference in the DNA content of the transfected cells was observed (data not shown). Furthermore, the increase in TGF- $\beta 1$ protein by excess wild-type GFAT $(52.7 \pm 8 \mathrm{pg} / \mu \mathrm{g}$ DNA vs $38.2 \pm 4$ in control cells) was reduced to that of control by addition of the GFAT enzyme inhibitors azaserine (5 $\mu \mathrm{mol} / \mathrm{l})$ or 6-diazo-5-oxonorleucine $(20 \mu \mathrm{mol} / \mathrm{l})$ (39.3 \pm 13 and $36.7 \pm 12 \mathrm{pg} / \mu \mathrm{g}$ DNA, respectively).

To assess whether the increased TGF- $\beta 1$ expression caused by enhanced GFAT activity results in increased matrix protein production, we measured the concentration of fibronectin in the supernatant. The increased TGF- $\beta 1$ protein expression of the mesangial cells transfected with wild-type GFAT expression vector is accompanied by an accumulation of fibronectin protein (Fig. 2B). Quantitative determination by ELISA showed an 1.5-fold production of fibronectin. Again, no difference between transfection with the empty expression vector and mutated GFAT ex-

\section{TGF-81 protein}
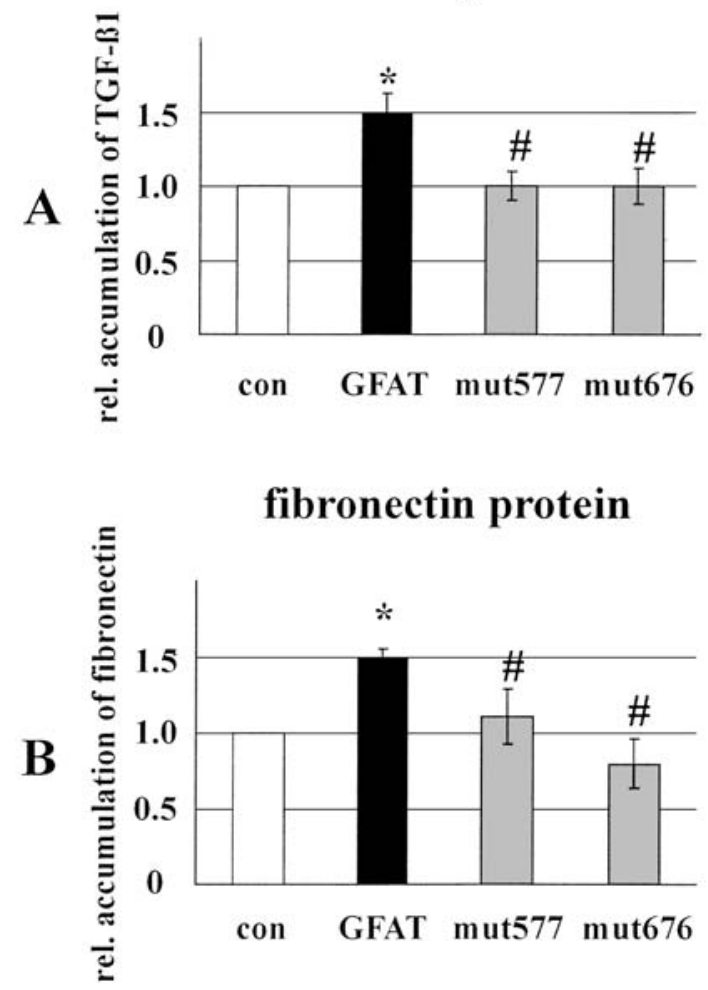

Fig. 2A, B. Effect of GFAT overexpression on TGF- $\beta 1$ and fibronectin expression in mesangial cells. Mesangial cells were transfected with wild-type and mutated GFAT expression vectors and the concentration of secreted TGF- $\beta 1$ or fibronectin protein was measured by ELISA $48 \mathrm{~h}$ after transfection. (A) Relative accumulation of TGF- $\beta 1$ in the supernatant of the transfected cells. Data are expressed as relative amount of TGF- $\beta 1$ protein/ $\mu \mathrm{g}$ of total DNA compared to control cells. (B) Relative accumulation of fibronectin measured by ELISA. Data are expressed as relative amount of fibronectin protein/ $\mu \mathrm{g}$ of total DNA compared to control cells. Results shown are means \pm SEM of three independent experiments. $* p<0.05$ wild-type vs. control, \# $p<0.05$ mutant vs. wild-type

pression vectors was observed. Similar results were obtained when fibronectin production was assessed by western blotting (data not shown). These data indicate that only the enhanced expression of functional GFAT protein, but not of the mutated forms, leads to an increase in TGF- $\beta 1$ and fibronectin production in mesangial cells.

\section{Discussion}

In these experiments we provide further causal evidence for a participation of the hexosamine biosynthetic pathway in the development of diabetic kidney disease by upregulation of TGF- $\beta 1$ gene expression. The overexpression of GFAT, the rate-limiting enzyme of this pathway, leads to an activation of this pathway [9] and subsequently induces TGF- $\beta 1$ protein expression and furthermore the expression of 
the extracellular matrix protein fibronectin in mesangial cells. Since this data together with our previous results, which showed that GFAT inhibition with azaserine prevents the high glucose-induced TGF- $\beta 1$ expression in mesangial cells [7], show that only expression of enzymatically active GFAT protein is effective we conclude that increased flux through the hexosamine pathway led to elevated TGF- $\beta 1$ gene expression.

Moreover, we showed the importance of amino acid 577 and 676 of GFAT for its enzymatic activity. While lysine 676 is likely to participate in the formation of a Schiff' base with fructose-6-phosphate, histidine 577 catalyses the ring-opening step. Both mutations resulted in a complete loss of GFAT enzyme activity.

In conclusion, our data show that enhanced GFAT activity in mesangial cells in ambient normo-physiologically glucose concentrations is sufficient for subsequent increased production of TGF- $\beta 1$ and fibronectin, thereby mimicking the effect of high glucose on TGF- $\beta 1$ expression. Our result supports previous suggestions that enhanced flux through the hexosamine pathway could increase the activity of transcription factors by O-GlcNAc-modification and subsequent synthesis of cytokines [2].

Acknowledgements. We gratefully acknowledge the donation of drosophila GFAT protein from H.-R. Graack, Berlin. The work was supported by the Deutsche Forschungsgemeinschaft (Schl 239-6) to E.S.

\section{References}

1. Mc Knight GL, Mudri SL, Mathewes SL et al. (1992) Molecular cloning, cDNA sequence, and bacterial expression of human glutamine:fructose-6-phosphate amidotransferase. J Biol Chem 267:25208-25212

2. Comer FI, Hart GW (2000) O-glycosylation of nuclear and cytosolic proteins. J Biol Chem 275:29179-29182

3. Marshall S, Bacote V, Traxinger RR (1991) Discovery of a metabolic pathway mediating glucose-induced desensitization of the glucose transport system. Role of hexosamine biosynthesis in the induction of insulin resistance. J Biol Chem 266:4706-4712

4. Rossetti MD (2000) Perspective: hexosamines and nutrient sensing. Endocrinology 141:1922-1925

5. Obici S, Wang J, Chowdury R et al. (2002) Identification of a biochemical link between energy intake and energy expenditure. J Clin Invest 109:1599-1605

6. McClain DA, Paterson AJ, Roos MD, Wie X, Kudlow JE (1992) Glucose and glucosamine regulate growth factor gene expression in vascular smooth muscle cells. Proc Natl Acad Sci USA 89:8150-8154

7. Kolm-Litty V, Sauer U, Nerlich A, Lehmann R, Schleicher ED (1998) High glucose-induced transforming growth factor $\beta 1$ production is mediated by the hexosamine pathway in porcine glomerular mesangial cells. J Clin Invest 101:160-169

8. Ziyadeh FN, Sharma K, Ericksen M, Wolf G (1994) Stimulation of collagen gene expression and protein synthesis in murine mesangial cells by high glucose is mediated by autocrine activation of transforming growth factor-beta. J Clin Invest 93:536-542

9. Weigert C, Brodbeck K, Lehmann R, Haering HU, Schleicher ED (2001) Overexpression of glutamine:fructose-6-phosphate-amidotransferase induces transforming growth factor- $\beta 1$ synthesis in NIH-3T3 fibroblasts. FEBS Lett 488:95-99

10. Nerlich AG, Sauer U, Kolm-Litty V, Wagner E, Koch M, Schleicher ED (1998) Expression of glutamine:fructose-6phosphate amidotransferase in human tissues: Evidence for high variability and distinct regulation in diabetes. Diabetes $47: 170-178$ 